\title{
SARS-CoV-2-related thyroid disorders: a synopsis for nuclear medicine thyroidologists
}

\author{
Luca Giovanella ${ }^{1,2,3}$ - Rosaria M. Ruggeri ${ }^{4}$ Petra Petranović Ovčariček ${ }^{3,5}$ - Alfredo Campenni ${ }^{3,6}$. \\ Giorgio Treglia $^{1,7,8,9} \cdot$ Desiree Deandreis ${ }^{3,10}$
}

Published online: 25 March 2021

(C) The Author(s), under exclusive licence to Springer-Verlag GmbH Germany, part of Springer Nature 2021

The coronavirus disease-19 (COVID-19) is an infectious disease caused by the enveloped RNA $\beta$-severe acute respiratory syndrome-coronavirus-2 (SARS-CoV-2). The COVID-19 may have a variable presentation, from an asymptomatic disease to acute respiratory distress syndrome (ARDS) and multi-organ failure. Impairment of endocrine systems may also occur in COVID-19 patients and thyroid gland involvement was reported in a not negligible number of patients, as documented in several studies since the pandemic outbreak. Abnormal thyroid function tests (TSH and/or thyroid hormones) are frequently reported in COVID-19 patients with variable prevalence and mild to moderate severity in available studies, as recently reviewed by our group [1]. In addition, to provide thyroid imaging and radioiodine therapy, nuclear medicine physicians are also involved, especially in Europe, in clinical patients' management. Then, being aware of the multiple and complex interactions between SARS$\mathrm{CoV}-2$ infection and thyroid function is relevant to nuclear medicine physicians in order to provide adequate and high-quality diagnosis, treatment, and follow-up.

This article is part of the Topical Collection on Endocrinology

Luca Giovanella luca.giovanella@eoc.ch

1 Clinic for Nuclear Medicine and Competence Centre for Thyroid Diseases, Imaging Institute of Southern Switzerland, Ente Ospedaliero Cantonale, Via A. Gallino 12, 6500 Bellinzona, Switzerland

2 Clinic for Nuclear Medicine, University Hospital and University of Zurich, Zurich, Switzerland

3 Thyroid Committee, European Association of Nuclear Medicine, Vienna, Austria

4 Unit of Endocrinology, Department of Clinical and Experimental Medicine, University of Messina, Messina, Italy

\section{SARS-CoV-2 effects on thyroid cells}

Two main mechanisms account for thyroid function perturbations in COVID-19 patients: (1) a viral effect on target cells and (2) an indirect effect of systemic inflammatory immune response (Fig. 1). A third condition, the so-called euthyroid sick syndrome (ESS), is typically observed in severely ill patients and represents an adaptive mechanism rather than a true thyroid dysfunction. Then, discriminating ESS from direct or indirect SARS-CoV-2 effects on thyroid function is relevant to avoid inappropriate treatments.

\section{Destructive thyroiditis}

Destructive thyroiditis usually manifests with initial thyrotoxicosis (1-2 months) followed by hypothyroidism (1-3 months) and, in most cases, a final restoration of euthyroidism [1]. Several cases of destructive thyroiditis are reported in COVID-19 patients, which is in line with the well-known association between many respiratory viruses and destructive thyroiditis (i.e., De Quervain's thyroiditis) $[1,2]$. It is

5 Department of Oncology and Nuclear Medicine, University Hospital Center "Sestre milosrdnice", Zagreb, Croatia

6 Unit of Nuclear Medicine, Department of Biomedical and Dental Sciences and Morpho-Functional Imaging, University of Messina, Messina, Italy

7 Faculty of Biology and Medicine, University of Lausanne, Lausanne, Switzerland

8 Faculty of Biomedical Sciences, Università della Svizzera italiana, Lugano, Switzerland

9 Academic Education, Research and Innovation Area, Ente Ospedaliero Cantonale, Bellinzona, Switzerland

10 Nuclear Medicine Division, Department of Medical Sciences, AOU Città della Salute e della Scienza, University of Turin, Turin, Italy 
Fig. 1 Direct and indirect effects of SARS-CoV-2 on thyroid cells and the hypothalamus-pituitarythyroid axis

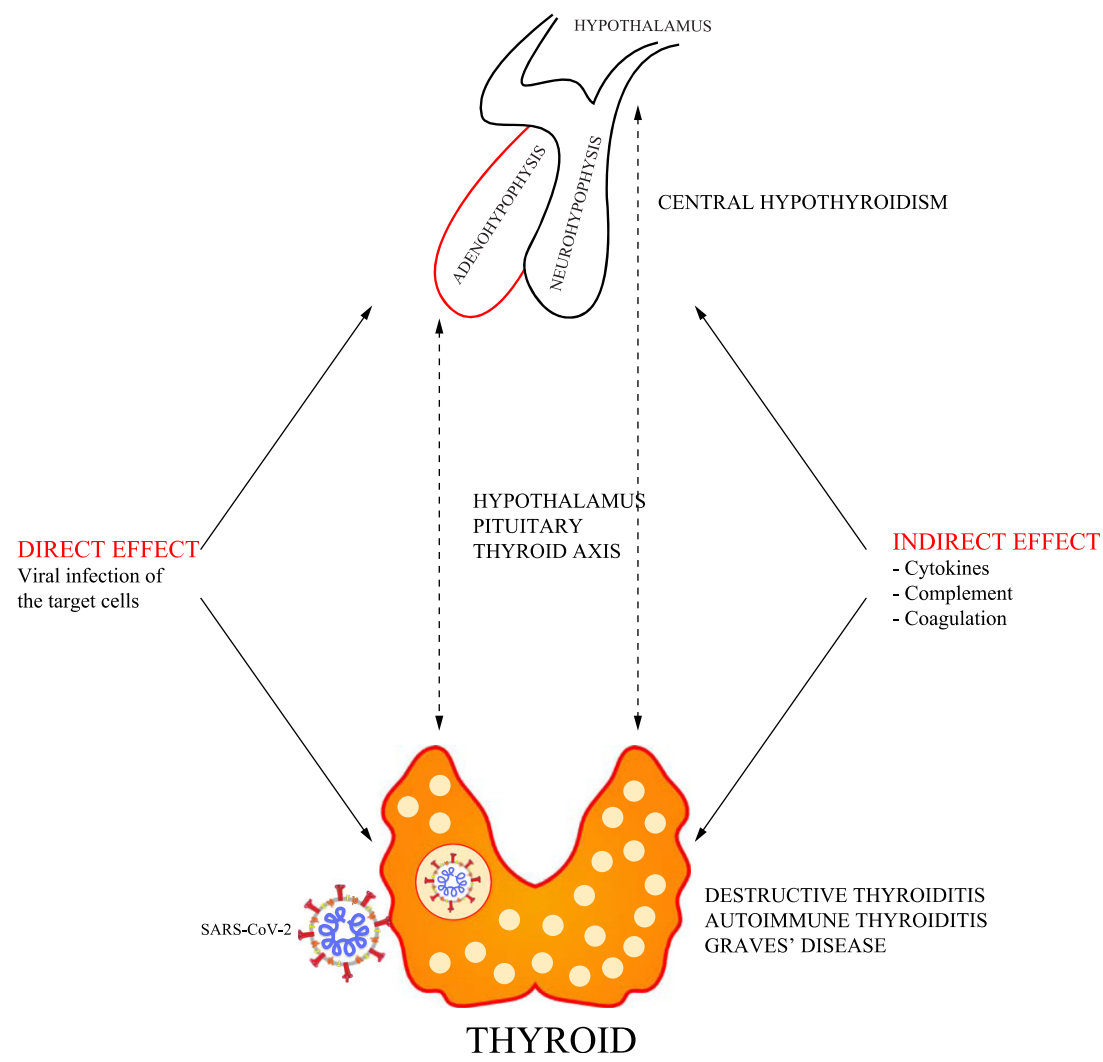

generally postulated that viral infections lead to necrosis or apoptosis of the follicular cells, which is followed by the release of antigens, the formation of altered antigens, secretion of cytokines and chemokines, altered HLA-DR expression, and activation of toll-like receptors [3].

The SARS-CoV-2 spike proteins bind to angiotensinconverting enzyme 2 (ACE2) receptors which are present on the surface of epithelial cells. Genes encoding ACE2 receptors are variably expressed in human organs, with the highest expression in the small intestine, testis, heart, thyroid, kidney, and lungs [4]. Then, the high expression of ACE2 receptors in thyroid cells may explain direct effects on thyroid parenchyma due to the SARS-CoV-2 entrance in follicular thyroid cells via the ACE2 receptors and transmembrane protease serine 2 (TMPRSS2) [5]. Available autoptic studies found cases with no significant damage of follicular thyroid cells in some cases, and chronic inflammation of the thyroid, and follicular epithelial cell disruption or interstitial lymphocytic infiltration in others, respectively. Additionally, no SARSCoV-2 was found in thyroid tissues by immunohistochemistry and polymerase chain reaction analysis $[6,7]$. These studies, however, are limited, not specifically focused on thyroid disorders, and notably, clinical data and thyroid hormone profile of these patients have not been adequately reported. Clinical studies are more consistent and data are accumulating on thyrotoxicosis and destructive thyroiditis in COVID-19 patients. Lania and colleagues reported a status of thyrotoxicosis (overt in about half of cases) in 58 of 287 COVID-19 patients $(20.2 \%)$ in absence of anti-thyroid antibodies and with an inverse correlation between TSH values and interleukin-6 (IL-6) [8]. Muller et al. [9] assessed the prevalence of thyrotoxicosis, suggestive of destructive thyroiditis, in 93 and 101 patients admitted to intensive care unit (HICU) due to COVID-19 or noninfectious conditions and 52 COVID-19 patients admitted to the low-intensity care unit. Among patients admitted to HICU, the prevalence of destructive thyroiditis was estimated at $10 \%$ in COVID-19 patients as compared to $0.5 \%$ in notCOVID-19 ones. Additionally, lower serum TSH levels and higher C-reactive protein levels were found in HICU patients with COVID-19 compared to those without and COVID-19 patients admitted to the low-intensity care unit, respectively. In addition, several cases of subacute painful thyroiditis or painless/silent thyroiditis have been diagnosed in COVID-19 patients (either with asymptomatic/mild or moderate/severe forms, respectively) that were reported in the literature $[1,10$, 11]. From the clinical point of view, it is important to emphasize that more than $50 \%$ of patients presented with overt thyrotoxicosis that may potentially worsen the underlying disease and complicate the recovery phase $[8,9,12]$.

\section{SARS-CoV-2-triggered autoimmune thyroid diseases}

Compared to the frequent destructive thyrotoxicosis, only four cases of Graves' disease were reported until now. Three patients had a previous history of Graves' disease and relapsed during (1 case) or few months after COVID-19 infection (2 
cases). The fourth patient had no history of previous thyroid diseases and developed Graves' disease 2 months after COVID-19 [13, 14]. Overall, such anecdotal data implicate SARS-CoV-2 infection as a potential driver for new cases or recurrences of Graves' disease. Autoimmune hypothyroidism occurs less frequently during or after COVID-19. Lania et al. found primary hypothyroidism in 5.2\% (15/287) of COVID19 patients (10\% of overt and $90 \%$ of subclinical hypothyroidism) [8]. It can represent the second, generally transient, phase of destructive thyroiditis (see above). Alternatively, indirect viral damage via pro-inflammatory cytokine storm and triggered autoimmune reaction was also hypothesized. Even if available literature is still insufficient to prove the association between COVID-19 and autoimmune diseases, the development of cytokine release syndrome and the triggering of autoimmunity support this hypothesis [5].

\section{SARS-CoV-2 effects on the hypothalamus-pituitary-thyroid axis}

Central hypothyroidism was observed in 2-6\% of patients hospitalized for non-mild COVID-19 [7, 8]. Direct injury may be a result of viral infection of the pituitary and hypothalamus, while an indirect injury is a consequence of aberrant immune response, subsequently affecting coagulation, complement system, and cytokines. Central hypothyroidism is characterized by low FT4 in combination with low to normal TSH levels and, fortunately, it appears to be reversible, as TSH and FT4 normalized after COVID-19 recovery in cases reported in the literature.

\section{Euthyroid sick syndrome}

The hallmark of the ESS is low FT3, with or without low FT4, in combination with normal or low TSH in clinically euthyroid patients with non-thyroidal systemic illness. Furthermore, it was already demonstrated that in the prolonged phase of critical illness, ESS is associated with adverse outcomes: accordingly, ESS was more frequent in the deceased compared to recovered patients with severe or critical COVID-19 [15].

\section{Cross-talk between different mechanisms}

Patients with COVID-19 may be affected by a combination of the above-mentioned pathways; i.e., the direct and indirect effects on target cells (thyroid, pituitary, and hypothalamus) and ESS may intertwine and give paradoxical results of hormonal status. For example, a combination of destructive thyroiditis and ESS may result in TSH suppression and increased FT4, but decreased FT3, which was defined as "low-T3 toxicosis" [8]. The same situation is theoretically possible in the case of simultaneous manifestation of ESS and less frequently observed Graves' disease. Careful evaluation and accurate differential diagnosis are pivotal here to appropriately treat true thyroid dysfunctions and avoid inappropriate treatment in patients with adaptive modifications of thyroid hormones constellation or either in "in vivo" (i.e., drugs) or "in vitro" interferences.

\section{Approaching thyroid dysfunctions in COVID-10 patients}

Although assessment of thyroid function for COVID-19 patients is still not recommended by the World Health Organization, thyroid function testing is mandatory in patients with known thyroid dysfunctions and those with symptoms suggesting a thyroid disease. Baseline thyroid function testing can be considered in COVID-19 patients, especially those having intermediate to severe illness, to prompt detection and (if needed) treatment of thyroid dysfunctions. Depending on the results of initial thyroid function laboratory testing, further diagnostic procedures may be required to discriminate causes of hormone perturbations.

\section{Thyrotoxicosis}

Thyrotoxicosis may negatively impact COVID-19 course by increasing the cardiovascular complications (including myocarditis, acute myocardial infarction, heart failure, arrhythmias, and venous thromboembolic events) and contribute to increasing the COVID-19-related mortality, especially in patients with severe disease $[6,12]$. The differential diagnosis of thyrotoxicosis is based on clinical examination and determination of hormonal status. Recently, the Endocrine Society/ American Association of Clinical Endocrinologists underlined that thyroid scintigraphy, not ultrasound, should be used to assess the etiology of the thyrotoxicosis and advised against requiring thyroid ultrasound in patients with abnormal thyroid function tests if there is no palpable abnormality of the thyroid gland (https://www.choosingwisely.org/ clinician-lists/endocrine-society-thyroid-ultrasounds-inpatients-with-abnormal-thyroid-function-tests/). Accordingly, thyroid scintigraphy should be suggested as a first-line procedure in COVID-19 patients with biochemically confirmed thyrotoxicosis to rapidly discriminate destructive thyroiditis from the rare cases of Graves' disease and to rapidly provide appropriate treatment (i.e., non-steroidal anti-inflammatory drugs or steroids for destructive thyroiditis and anti-thyroid drugs for Graves' disease) (Fig. 2). This approach is easily applied to de-isolated outpatients and, with adequate protocols, in-hospital patients with mild to moderate disease; 


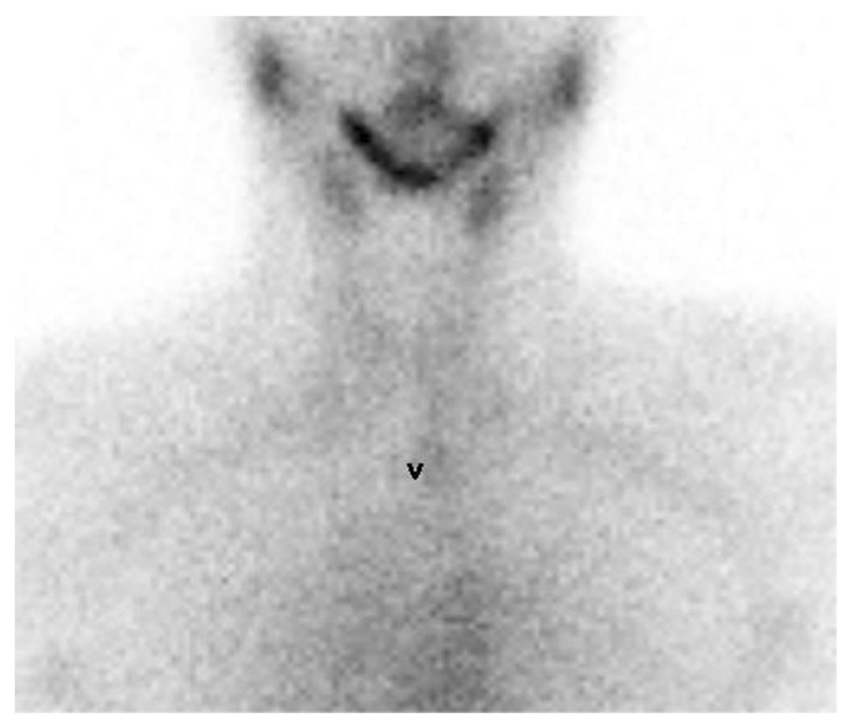

Fig. $299 \mathrm{mTc}$-pertechnetate thyroid scintigraphy demonstrating destructive thyroiditis in a 35-year-old female with SARS-CoV-2related thyrotoxicosis $(\mathrm{TSH}<0.01 \mathrm{mUI} / \mathrm{L}(0.27-4.2 \mathrm{mUI} / \mathrm{L}), \mathrm{FT} 4$ $32.5 \mathrm{pmol} / \mathrm{L}(12.0-22.0 \mathrm{pmol} / \mathrm{L})$, FT3 $7.1 \mathrm{pmol} / \mathrm{L}(3.1-6.8))$

however, the access to thyroid scintigraphy could be limited in intensive care units. In such cases, surrogate markers can be used: an increased FT4/FT3 ratio, a negative TSH-receptor antibody titer and a sustained increase of serum thyroglobulin are all related to follicular destruction [16]. In addition, when available, portable hand-held gamma cameras may allow the estimation of thyroid uptake at the patient's bedside [17].

\section{Euthyroid sick syndrome and "low T3-(pseudo)thyrotoxicosis"}

Significantly lower serum concentrations of FT3 were found in patients with severe COVID-19 than in non-severely ill patients and independently predicted all-cause mortality of patients with severe COVID-19 [15]. Interestingly, no significant relationship was found between all-cause mortality and TSH, FT4, and FT3/FT4 ratio in the multivariate analysis. Accordingly, FT3 may become a simple tool for stratified management of patients with severe COVID-19 [8]. Notably, although the clinical significance of ESS is unclear, the use of thyroxine for patients with ESS did not produce additional benefits and is discouraged. Interestingly, severely ill patients presenting with reduced TSH and increased FT4 but low FT3 levels were described. A "low-T3 thyrotoxicosis," due to an increased T3 to reverse-T3 deiodination in such patients, was hypothesized [7, 8]. However, it should be noted that steroids and heparin, commonly used in such patients, can interfere with both parameters. Serum TSH reduction is observed after steroid administration while fractionated or unfractionated heparin may produce an artifactual elevation in measured FT4 concentrations likely due to displacement of thyroid hormones from their binding sites by free fatty acids liberated in vitro [18]. A further point in favor of such hypothesis is represented by prompt normalization of thyroid function test after recovery from the critical phase while longer recoveries are observed after follicular destructive damage occurs.

\section{Hypothyroidism}

Relatively few cases of hypothyroidism were reported in COVID-19 patients and a similar prevalence was demonstrated by comparing two series of $126(5.6 \%)$ and $287(5.2 \%)$ hospitalized COVID-19 patients [7, 8]. There is insufficient data to determine if autoimmune hypothyroidism preceded the COVID-19 or was triggered by the infection. However, the known association among COVID-19, the development of cytokine release syndrome, and the triggering of autoimmunity supports the hypothesis of COVID-19-related hypothyroidism [19]. Available hypothyroidism clinical guidelines can be followed in managing such relatively rare patients.

\section{Conclusions}

In conclusion, altered thyroid function is not infrequently observed in COVID-19 patients. Prompt detection and accurate discrimination are required to treat conditions related to worse prognosis and avoid inappropriate therapies in other cases. Good knowledge of different pathophysiological mechanisms and their cross-talk is needed to carefully evaluate thyroid function tests. Thyroid scintigraphy, whenever feasible, may consistently contribute to clarify doubtful cases. Finally, low FT3 levels are independently associated with increased allcause mortality and may serve as a surrogate prognostic biomarker.

\section{Declarations}

Informed consent Not applicable

Ethical approval Not applicable

Conflict of interest LG is a member of the Roche Diagnostics advisory board and has received research grants and speaker honoraria from Roche Diagnostics, IBSA, and Sanofi-Genzyme. Other authors declare no conflicts of interest.

\section{References}

1. Giovanella L, Ruggeri RM, Ovčariček PP, Campenni A, Treglia G, Deandreis D. Prevalence of thyroid dysfunction in patients with 
COVID-19: a systematic review. Clin Transl Imaging. 2021;1-8. https://doi.org/10.1007/s40336-021-00419-y.

2. Slatosky J, Shipton B, Wahba H. Thyroiditis: differential diagnosis and management. Am Fam Physician. 2000;61:1047-52.

3. Desailloud R, Hober D, Desailloud R. Viruses and thyroiditis: an update. Virol J. 2009;6:5. https://doi.org/10.1186/1743-422X-6-5.

4. Hoffmann M, Kleine-Weber H, Schroeder S, Krüger N, Herrler T, Erichsen S, et al. SARS-CoV-2 cell entry depends on ACE2 and TMPRSS 2 and is blocked by a clinically proven protease inhibitor. Cell. 2020;181:271-80.

5. Rotondi M, Coperchini F, Ricci G, Denegri M, Croce L, Ngnitejeu $\mathrm{ST}$, et al. Detection of SARS-COV-2 receptor ACE-2 mRNA in thyroid cells: a clue for COVID-19-related subacute thyroiditis. J Endocrinol Investig. 2020;6:1-6. https://doi.org/10.1007/s40618020-01436-w.

6. Chen T, Wu D, Chen H, Yan W, Yang D, Chen G, et al. Clinical characteristics of 113 deceased patients with coronavirus disease 2019: retrospective study. BMJ. 2020;368:m1091. https://doi.org/ 10.1136/bmj.m1091.

7. Hanley B, Naresh KN, Roufosse C, Nicholson AG, Weir J, Cooke GS, et al. Histopathological findings and viral tropism in UK patients with severe fatal COVID-19: a post-mortem study. Lancet Microb. 2020;1(6):e245-53. https://doi.org/10.1016/S26665247(20)30115-4.

8. Lania A, Sandri MT, Cellini M, Mirani M, Lavezzi E, Mazziotti G. Thyrotoxicosis in patients with COVID-19: the THYRCOV study. Eur J Endocrinol. 2020;183:381-7.

9. Muller I, Cannavaro D, Dazzi D, Covelli D, Mantovani G, Muscatello A, et al. SARS-CoV-2-related atypical thyroiditis. Lancet Diabetes Endocrinol. 2020;8:739-41.

10. Ruggeri RM, Campennì A, Siracusa M, Frazzetto G, Gullo D. Subacute thyroiditis in a patient infected with SARS-COV-2: an endocrine complication linked to the COVID-19 pandemic.
Hormones (Athens). 2020:1-3. https://doi.org/10.1007/s42000020-00230-w.

11. Khatri A, Charlap E, Kim A. Subacute thyroiditis from COVID-19 infection: a case report and review of literature. Eur Thyroid J. 2020. https://doi.org/10.1159/000511872.

12. Madjid M, Safavi-Naeini P, Solomon SD, Vardeny O. Potential effects of coronaviruses on the cardiovascular system: a review. JAMA Cardiol. 2020;5:831-40.

13. Mateu-Salat M, Urgell E, Chico A. SARS-COV-2 as a trigger for autoimmune disease: report of two cases of Graves' disease after COVID-19. J Endocrinol Investig. 2020;43(10):1527-8.

14. Jiménez-Blanco S, Pla-Peris B, Marazuela M. COVID-19: a cause of recurrent Graves' hyperthyroidism? J Endocrinol Investig. 2020;6:1-2. https://doi.org/10.1007/s40618-020-01440-0.

15. Zou R, Wu C, Zhang S, Wang G, Zhang Q, Yu B, et al. Euthyroid sick syndrome in patients with COVID-19. Front Endocrinol (Lausanne). 2020;11:566439.

16. Pearce EN, Farwell AP, Braverman LE. Thyroiditis. N Engl J Med. 2003;348:2646-55.

17. Duch J. Portable gamma cameras: the real value of an additional view in the operating theatre. Eur J Nucl Med Mol Imaging. 2011;38:633-5.

18. Koulouri O, Moran C, Halsall D, Chatterjee K, Gurnell M. Pitfalls in the measurement and interpretation of thyroid function tests. Best Pract Res Clin Endocrinol Metab. 2013;27:745-62.

19. Tee LY, Harjanto S, Rosario BH. COVID-19 complicated by Hashimoto's thyroiditis. Singap Med J. 2020. https://doi.org/10. 11622/smedj.2020106.

Publisher's note Springer Nature remains neutral with regard to jurisdictional claims in published maps and institutional affiliations. 